ALEA, Lat. Am. J. Probab. Math. Stat. 14, 201-218 (2017)

DOI: 10.30757/ALEA.v14-12

\title{
Reflected BSDEs when the obstacle is not right-continuous in a general filtration
}

\author{
Brahim Baadi and Youssef Ouknine \\ Ibn Tofaïl University, \\ Department of mathematics, faculty of sciences, \\ BP 133, Kénitra, Morocco \\ E-mail address: baadibrahim@gmail.com \\ Cadi Ayyad University, \\ Av. Abdelkrim Khattabi \\ 40000, Guéliz Marrakesh, Morocco, and \\ Hassan II Academy of Sciences and Technology, Rabat, Morocco \\ E-mail address: ouknine@uca.ma
}

\begin{abstract}
We prove existence and uniqueness of the reflected backward stochastic differential equation's (RBSDE) solution with a lower obstacle which is assumed to be right upper-semicontinuous but not necessarily right-continuous in a filtration that supports a Brownian motion $W$ and an independent Poisson random measure $\pi$. The result is established by using some tools from the general theory of processes such as Mertens decomposition of optional strong (but not necessarily right continuous) supermartingales and some tools from optimal stopping theory, as well as an appropriate generalization of Itô's formula due to Gal'čuk and Lenglart. Two applications on dynamic risk measure and on optimal stopping will be given.
\end{abstract}

\section{Introduction}

The notion of Backward Stochastic Differential Equations (BSDEs in short) was introduced by Bismut $(1973,1976)$ in the case of a linear driver. The nonlinear case was developed by Pardoux and Peng $(1990,1992)$. BSDEs have found a number of applications in finance, that is pricing and hedging of European options and recursive utilities (for instance El Karoui et al., 1997b).

Reflected Backward Stochastic Differential Equations (RBSDEs in short) have been introduced by El Karoui et al. (1997a) and were useful, for example, in the

Received by the editors May 6th, 2016; accepted March 3rd, $201 \%$.

2010 Mathematics Subject Classification. 60K35, 82B43.

Key words and phrases. Backward stochastic differential equation, reflected backward stochastic differential equation, general filtration, strong optional supermartingale, Mertens decomposition. 
study of American option. The difference between the two types of equations (BSDEs and RBSDEs) is that the second can be seen as a variant of the first in which the first component of the solution is constrained to remain greater than or equal to a given process called obstacle or barrier, and there is an additional nondecreasing predictable process which keeps the first component of the solution above the obstacle. The work of El Karoui et al. (1997a) considers the case of a Brownian filtration and a continuous obstacle. After there have been several extensions of this work to the case of a discontinuous obstacle, for example, Hamadène (2002), Hamadène and Ouknine (2003, 2016), Essaky (2008) and Crépey and Matoussi (2008) etc.

The right continuity of the obstacle is the difference between these extensions and the paper of Grigorova et al. (2015). In this work, the authors present a further extension of the theory of RBSDEs to the case where the obstacle is not necessarily right-continuous in a Brownian filtration.

In the present paper, we generalize the result of uniqueness and existence of the RBSDE's solution in Grigorova et al. (2015) to the case of a larger stochastic basis, i.e. in a filtration that supports a Brownian motion $W$ and an independent Poisson random measure $\pi$, we establish existence and uniqueness of solutions, in appropriate Banach spaces, to the following RBSDE:

$$
\begin{array}{r}
Y_{\tau}=\xi_{T}+\int_{\tau}^{T} f\left(s, Y_{s}, Z_{s}, \psi_{s}\right) \\
d s-\int_{\tau}^{T} Z_{s} d W_{s}-\int_{\tau}^{T} \int_{\mathcal{U}} \psi_{s}(u) \widetilde{\pi}(d u, d s)-\int_{\tau}^{T} d M_{s} \\
+A_{T}-A_{\tau}+C_{T-}-C_{\tau-} \text { for all } \tau \in \mathcal{T}_{0, T} .
\end{array}
$$

The solution is given by $(Y, Z, \psi, M, A, C)$, where $M$ is an orthogonal local martingale. We assume that the function $f$ is Lipschitz with respect to $y, z$ and $\psi$. To prove our results we use tools from the general theory of processes such as Mertens decomposition of strong optional (but not necessarily right-continuous) supermartingales (generalizing Doob-Meyer decomposition) and some tools from optimal stopping theory, as well as a generalization of Itô's formula to the case of strong optional (but not necessarily right-continuous) semimartingales due to Gal'čuk (1980) and Lenglart (1980).

We recover these natural differential equations by studying the limit of a system of reflected BSDEs

$$
\left\{\begin{aligned}
& Y_{\tau}^{n}= \xi_{T}+\int_{\tau}^{T} f\left(s, Y_{s}^{n}, Z_{s}^{n}, \psi_{s}^{n}\right) d s+K_{T}^{n}-K_{\tau}^{n}-\int_{\tau}^{T} Z_{s}^{n} d W_{s} \\
& \quad-\int_{\tau}^{T} \int_{\mathcal{U}} \psi_{s}^{n}(u) \widetilde{\pi}(d u, d s)-\int_{\tau}^{T} d M_{s}^{n} \\
& Y_{\tau}^{n} \geq \xi_{\tau}
\end{aligned}\right.
$$

where $K_{t}^{n}=n \int_{0}^{t}\left(Y_{s}^{n}-\xi_{s}\right)^{-} d s$. Essaky (2008) proved, by a monotonic limit theorem, that $\left(Y^{n}, Z^{n}, K^{n}, \psi^{n}, M^{n}\right)$ has, in some sense, a limit $(Y, Z, K, \psi, M)$ which satisfies a reflected BSDE with $\xi$ a càdlàg barrier (see also Peng, 1999 for the case of filtration generated only by a Brownian motion).

It is well known that if $\xi$ is a càdlàg barrier then $Y$ is also a càdlàg process (Theorem 3.1 in Essaky, 2008 for filtration generated by a Brownian motion and Poisson point process, and Lemma 2.2 in Peng, 1999 for the Brownian filtration). But if the barrier $\xi$ is only optional the limit $Y$ of $Y^{n}$ is $\mathcal{E}^{f}$-super-martingale, then $Y$ has left and right limits (see Dellacherie and Meyer, 1980, Theorem 4 page 408).

In this sense, we know that $\left(Y^{n}, Z^{n}, \psi^{n}, M^{n}\right)$ converge to $(Y, Z, \psi, M)$ and the limit $K$ of $K_{t}^{n}=n \int_{0}^{t}\left(Y_{s}^{n}-\xi_{s}\right)^{-} d s$ is a làdlàg process that can be written as 
$K=A+C_{-}$where $A$ an increasing càdlàg predictable process satisfying $A_{0}=0$, $E\left(A_{T}\right)<\infty$, and $C$ an increasing càdlàg optional process and $E\left(C_{T}\right)<\infty$.

The paper is decomposed as follows: in the second section, we give the mathematical setting (preliminary, definitions and properties). In subsection 2.1 we recall the change of variables formula for optional semimartingales which are not necessarily right continuous (Gal'čuk-Lenglart decomposition for strong optional semimartingales). In the third section, we define our RBSDE and we prove existence and uniqueness of the solution in a general filtration. In the last section, we give two applications of reflected BSDEs where the right-continuity of the obstacle is not necessarily used: application on dynamic risk measure and on optimal stopping.

\section{Preliminaries}

Let $T>0$ be a fixed positive real number. Let us consider a filtered probability space $\left(\Omega, \mathcal{F}, \mathbb{P}, \mathbb{F}=\left\{\mathcal{F}_{t}, t \geq 0\right\}\right)$. The filtration is assumed to be complete, right continuous and quasi-left continuous, which means that for every sequence $\left(\tau_{n}\right)$ of $\mathbb{F}$ stopping times such that $\tau_{n} \nearrow \tau$ for some stopping time $\tau$ we have $\bigvee_{n \in \mathbb{Z}_{+}} \mathcal{F}_{\tau_{n}}=\mathcal{F}_{\tau}$. We assume that $\left(\Omega, \mathcal{F}, \mathbb{P}, \mathbb{F}=\left\{\mathcal{F}_{t}, t \geq 0\right\}\right)$ supports a $k$-dimensional Brownian motion $W$ and a Poisson random measure $\pi$ with intensity $\mu(d u) d t$ on the space $\mathcal{U} \subset \mathbb{R}^{m} \backslash\{0\}$. The measure $\mu$ is $\sigma$-finite on $\mathcal{U}$ such that

$$
\int_{\mathcal{U}}\left(1 \wedge|u|^{2}\right) \mu(d u)<+\infty
$$

The compensated Poisson random measure $\pi: \widetilde{\pi}(d u, d t)=\pi(d u, d t)-\mu(d u) d t$ is a martingale w.r.t. the filtration $\mathbb{F}$.

In this paper for a given $T>0$, we denote:

- $\mathcal{T}_{t, T}$ is the set of all stopping times $\tau$ such that $\mathbb{P}(t \leq \tau \leq T)=1$. More generally, for a given stopping time $\nu$ in $\mathcal{T}_{0, T}$, we denote by $\mathcal{T}_{\nu, T}$ the set of all stopping times $\tau$ such that $\mathbb{P}(\nu \leq \tau \leq T)=1$.

- $\mathcal{P}$ is the predictable $\sigma$-field on $\Omega \times[0, T]$ and

$$
\widetilde{\mathcal{P}}=\mathcal{P} \otimes \mathcal{B}(\mathcal{U})
$$

where $\mathcal{B}(\mathcal{U})$ is the Borelian $\sigma$-field on $\mathcal{U}$.

- $L^{2}\left(\mathcal{F}_{T}\right)$ is the set of random variables which are $\mathcal{F}_{T}$-measurable and squareintegrable.

- On $\widetilde{\Omega}=\Omega \times[0, T] \times \mathcal{U}$, a function that is $\widetilde{\mathcal{P}}$-measurable, is called predictable.

- $G_{l o c}(\pi)$ is the set of $\widetilde{\mathcal{P}}$-measurable functions $\psi$ on $\widetilde{\Omega}$ such that for any $t \geq 0$ a.s.

$$
\int_{0}^{t} \int_{\mathcal{U}}\left(\left|\psi_{s}(u)\right|^{2} \wedge\left|\psi_{s}(u)\right|\right) \mu(d u)<+\infty
$$

- $\mathbb{H}^{2, T}$ is the set of real-valued predictable processes $\phi$ such that

$$
\|\phi\|_{\mathrm{H}^{2, T}}^{2}=E\left[\int_{0}^{T}\left|\phi_{t}\right|^{2} d t\right]<\infty .
$$

- $\mathcal{M}_{\text {loc }}$ is the set of càdlàg local martingales orthogonal to $W$ and $\tilde{\pi}$ : if $M \in \mathcal{M}_{\text {loc }}$ then

$$
\left[M, W^{i}\right]_{t}=0, \quad 1 \leq i \leq k, \quad[M, \widetilde{\pi}(A)]_{t}=0
$$


for all $A \in \mathcal{B}(\mathcal{U})$.

- $\mathcal{M}$ is the subspace of $\mathcal{M}_{\text {loc }}$ of martingales.

As explained above, the filtration $\mathbb{F}$ supports the Brownian motion $W$ and the Poisson random measure $\pi$. We have the following lemma that we can find in Jacod and Shiryaev (2003, Chapter III, Lemma 4.24):

Lemma 2.1. Every local martingale $N$ has a decomposition

$$
N_{t}=\int_{0}^{t} Z_{s} d W_{s}+\int_{0}^{t} \int_{\mathcal{U}} \psi_{s}(u) \widetilde{\pi}(d u, d s)+M_{t}
$$

where $M \in \mathcal{M}_{l o c}, Z \in \mathbb{H}^{2, T}$ and $\psi \in G_{l o c}(\mu)$.

Now to define the solution of our reflected backward stochastic differential equation (RBSDE), let us introduce the following spaces:

- $\mathbb{S}^{2, T}$ is the set of real-valued optional processes $\phi$ such that:

$$
\||\phi|\|_{S^{2, T}}^{2}=E\left[\text { ess } \sup _{\tau \in \mathcal{T}_{0, T}}\left|\phi_{\tau}\right|^{2}\right]<\infty .
$$

- $\mathbb{M}^{2}$ is the subspace of $\mathcal{M}$ of all martingales such that:

$$
\|M\|_{\mathbb{M}^{2}}^{2}=E\left([M, M]_{T}\right)=E\left([M]_{T}\right)<+\infty .
$$

- $\mathbb{L}_{\pi}^{2}(0, T)=\mathbb{L}_{\pi}^{2}(\Omega \times(0, T) \times \mathcal{U})$ is the set of all processes $\psi \in G_{l o c}(\mu)$ such that:

$$
\|\psi\|_{\mathbb{L}_{\pi}^{2}}^{2}=E\left[\int_{0}^{T} \int_{\mathcal{U}}\left|\psi_{s}(u)\right|^{2} \mu(d u) d s\right]<+\infty .
$$

- $\mathbb{L}_{\mu}^{2}(0, T)=\mathbb{L}^{2}\left(\mathcal{U}, \mu ; \mathbb{R}^{d}\right)$ is the set of all measurable functions $\psi: \mathcal{U} \longrightarrow \mathbb{R}^{d}$ such that:

$$
\|\psi\|_{\mathbb{L}_{\mu}^{p}}^{2}=\int_{\mathcal{U}}|\psi(u)|^{2} \mu(d u)<+\infty
$$

- $\mathcal{E}^{2}(0, T)=\mathbb{S}^{2, T} \times \mathbb{H}^{2, T} \times \mathbb{L}_{\pi}^{2}(0, T) \times \mathbb{M}^{2} \times \mathbb{S}^{2, T} \times \mathbb{S}^{2, T}$.

The random variable $\xi$ is $\mathcal{F}_{T}$-measurable with values in $\mathbb{R}^{d}(d \geq 1)$ and $f$ : $\Omega \times[0, T] \times \mathbb{R}^{d} \times \mathbb{R}^{d \times k} \times \mathbb{L}_{\mu}^{2} \longrightarrow \mathbb{R}^{d}$ is a random function measurable with respect to $\operatorname{Prog} \times \mathcal{B}\left(\mathbb{R}^{d}\right) \times \mathcal{B}\left(\mathbb{R}^{d \times k}\right) \times \mathcal{B}\left(\mathbb{L}_{\mu}^{2}\right)$ where Prog denotes the $\sigma$-field of progressive subsets of $\Omega \times[0, T]$.

In the following we denote the spaces $\mathbb{H}^{2, T}$ and $\mathbb{S}^{2, T}$ by $\mathbb{H}^{2}$ and $\mathbb{S}^{2}$, as well as the norms $\|\cdot\|_{H^{2, T}}$ and $\left\||\cdot \||_{\mathbb{S}^{2, T}}\right.$ by $\| \cdot \|_{\mathbb{H}^{2}}$ and $\|\mid \cdot\|_{\mathbb{S}^{2}}$.

Definition 2.2. A function $f$ is said to be a driver if:

- $f: \Omega \times[0, T] \times \mathbb{R}^{2} \times \mathbb{L}_{\mu}^{2} \longrightarrow \mathbb{R}$ $(\omega, t, y, z, \psi) \longmapsto f(\omega, t, y, z, \psi)$ is $\mathcal{P} \otimes \mathcal{B}\left(\mathbb{R}^{2}\right) \otimes \mathcal{B}\left(\mathbb{L}_{\mu}^{2}\right)$-measurable.

- $E\left[\int_{0}^{T}|f(t, 0,0,0)|^{2} d t\right]<\infty$.

A driver $f$ is called a Lipschitz driver if moreover there exists a constant $K \geq 0$ such that $\mathbb{P} \otimes d t$-a.s., for each $\left(y_{1}, z_{1}, \psi_{1}\right)$ and $\left(y_{2}, z_{2}, \psi_{2}\right)$

$$
\left|f\left(\omega, t, y_{1}, z_{1}, \psi_{1}\right)-f\left(\omega, t, y_{2}, z_{2}, \psi_{2}\right)\right| \leq K\left(\left|y_{1}-y_{2}\right|+\left|z_{1}-z_{2}\right|+\left\|\psi_{1}-\psi_{2}\right\|_{\mathbb{L}_{\mu}^{2}}\right) \text {. }
$$

For a làdlàg process $\phi$, we denote by $\phi_{t+}$ and $\phi_{t-}$ the right-hand and left-hand limit of $\phi$ at $t$. We denote by $\Delta_{+} \phi_{t}=\phi_{t+}-\phi_{t}$ the size of the right jump of $\phi$ at $t$, and by $\Delta \phi_{t}=\phi_{t}-\phi_{t-}$ the size of the left jump of $\phi$ at $t$.

We give a useful property of the space $\mathbb{S}^{2}$ : 
Proposition 2.3. The space $\mathbb{S}^{2}$ endowed with the norm $\left\||\cdot \||_{\mathbb{S}^{2}}\right.$ is a Banach space.

Proof: The proof is given in Grigorova et al. (2015, Proposition 2.1).

The following proposition can be found in Nikeghbali (2006, Theorem 3.2.).

Proposition 2.4. Let $\left(X_{t}\right)$ and $\left(Y_{t}\right)$ be two optional processes. If for every finite stopping time $\tau$ one has, $X_{\tau}=Y_{\tau}$, then the processes $\left(X_{t}\right)$ and $\left(Y_{t}\right)$ are indistinguishable.

Let $\beta>0$. We will also use the following notation:

For $\phi \in \mathbb{H}^{2},\|\phi\|_{\beta}^{2}:=E\left[\int_{0}^{T} e^{\beta s} \phi_{s}^{2} d s\right]$. We note that on the space $\mathbb{H}^{2}$ the norms $\|\cdot\|_{\beta}$ and $\|\cdot\|_{\mathbb{H}^{2}}$ are equivalent.

For $\phi \in \mathbb{S}^{2}$, we define $\|\left.|\phi|\right|_{\beta} ^{2}:=E\left[\right.$ ess $\left.\sup _{\tau \in \mathcal{T}_{0, T}} e^{\beta \tau}\left|\phi_{\tau}\right|^{2}\right]$. We note that $\||\cdot|\|_{\beta}$ is a norm on $\mathbb{S}^{2}$ equivalent to the norm $\||\cdot|\|_{\mathbb{S}^{2}}$.

For $\phi \in \mathbb{L}_{\pi}^{2}$, the defined norm $\|\phi\|_{\mathbb{L}_{\pi}^{2, \beta}}=\sqrt{E\left[\int_{0}^{T} e^{\beta s} \int_{\mathcal{U}}\left|\phi_{s}(u)\right|^{2} \mu(d u) d s\right]}$ is equivalent to the norm $\|\phi\|_{\mathbb{L}_{\pi}^{2}}$ on $\mathbb{L}_{\pi}^{2}$.

For $M \in \mathbb{M}^{2}$, we have the equivalence between $\|M\|_{\mathbb{M}_{\beta}^{2}}=\sqrt{E\left[\int_{0}^{T} e^{\beta s} d[M]_{s}\right]}$ and $\|M\|_{\mathbb{M}^{2}}$ on $\mathbb{M}^{2}$.

2.1. Gal'čuk-Lenglart decomposition for strong optional semimartingales. In this section, we recall the change of variables formula for optional semimartingales which are not necessarily cad. The result can be seen as a generalization of the classical Itô formula and can be found in Gal'čuk (1980, Theorem 8.2) and Lenglart (1980, Section 3, page 538). We recall the result in our framework in which the underlying filtered probability space satisfies the usual conditions.

Theorem 2.5. (Gal'čuk-Lenglart) Let $n \in \mathbb{Z}_{+}$. Let $X$ be an $n$-dimensional optional semimartingale, i.e. $X=\left(X_{1}, \ldots, X_{n}\right)$ is an $n$-dimensional optional process with decomposition $X_{t}^{k}=X_{0}^{k}+N_{t}^{k}+A_{t}^{k}+B_{t}^{k}$, for all $k \in\{1, \ldots, n\}$ where $N_{t}^{k}$ is a (càdlàg) local martingale, $A_{t}^{k}$ is a right-continuous process of finite variation such that $A_{0}=0$ and $B_{t}^{k}$ is a left-continuous process of finite variation which is purely discontinuous and such that $B_{0-}=0$. Let $F$ be a twice continuously differentiable function on $\mathbb{R}^{n}$. Then, almost surely, for all $t \geq 0$,

$$
\begin{aligned}
F\left(X_{t}\right) & =F\left(X_{0}\right)+\sum_{k=1}^{n} \int_{] 0, t]} D^{k} F\left(X_{s-}\right) d\left(N^{k}+A^{k}\right)_{s} \\
& +\frac{1}{2} \sum_{k, l=1}^{n} \int_{] 0, t]} D^{k} D^{l} F\left(X_{s-}\right) d<N^{k c}, N^{l c}>_{s} \\
& +\sum_{0<s \leq t}\left[F\left(X_{s}\right)-F\left(X_{s-}\right)-\sum_{k=1}^{n} D^{k} F\left(X_{s-}\right) \Delta X_{s}^{k}\right] \\
& +\sum_{k=1}^{n} \int_{[0, t[} D^{k} F\left(X_{s}\right) d\left(B^{k}\right)_{s+} \\
& +\sum_{0 \leq s<t}\left[F\left(X_{s+}\right)-F\left(X_{s}\right)-\sum_{k=1}^{n} D^{k} F\left(X_{s}\right) \Delta_{+} X_{s}^{k}\right]
\end{aligned}
$$


where $D^{k}$ denotes the differentiation operator with respect to the $k$-th coordinate, and $N^{k c}$ denotes the continuous part of $N^{k}$.

Corollary 2.6. Let $Y$ be a one-dimensional optional semimartingale with decomposition $Y_{t}=Y_{0}+N_{t}+A_{t}+B_{t}$, where $N, A$ and $B$ are as in the above theorem. Let $\beta>0$. Then, almost surely, for all $t$ in $[0, T]$,

$$
\begin{aligned}
e^{\beta t} Y_{t}^{2} & =Y_{0}^{2}+\int_{0}^{t} \beta e^{\beta s} Y_{s}^{2} d s+2 \int_{0}^{t} e^{\beta s} Y_{s-} d(A+N)_{s} \\
& +\int_{0}^{t} e^{\beta s} d<N^{c}, N^{c}>_{s} \\
& +\sum_{0<s \leq t} e^{\beta s}\left(Y_{s}-Y_{s-}\right)^{2}+2 \int_{0}^{t} e^{\beta s} Y_{s} d(B)_{s+}+\sum_{0 \leq s<t} e^{\beta s}\left(Y_{s+}-Y_{s}\right)^{2} .
\end{aligned}
$$

Proof: For the corollary demonstration, it suffices to apply the change of variables formula from Theorem 2.5 with $n=2, F(x, y)=x y^{2}, X_{t}^{1}=e^{\beta t}$ and $X_{t}^{2}=Y_{t}$. Indeed, by applying Theorem 2.5 and by noting that the local martingale part and the purely discontinuous part of $X^{1}$ are both equal to 0 , we obtain

$$
\begin{aligned}
e^{\beta t} Y_{t}^{2} & =Y_{0}^{2}+\int_{0}^{t} \beta e^{\beta s} Y_{s}^{2} d s+2 \int_{0}^{t} e^{\beta s} Y_{s-} d(A+N)_{s} \\
& +\int_{0}^{t} e^{\beta s} d<N^{c}, N^{c}>_{s} \\
& +\sum_{0<s \leq t} e^{\beta s}\left(Y_{s}^{2}-Y_{s-}^{2}-2 Y_{s-}\left(Y_{s}-Y_{s-}\right)\right)+2 \int_{0}^{t} e^{\beta s} Y_{s} d(B)_{s+} \\
& +\sum_{0 \leq s<t} e^{\beta s}\left(Y_{s+}^{2}-Y_{s}^{2}-2 Y_{s}\left(Y_{s+}-Y_{s}\right)\right) .
\end{aligned}
$$

The desired expression follows as $\left(Y_{s}-Y_{s-}\right)^{2}=Y_{s}^{2}-Y_{s-}^{2}-2 Y_{s-}\left(Y_{s}-Y_{s-}\right)$ and $\left(Y_{s+}-Y_{s}\right)^{2}=Y_{s+}^{2}-Y_{s}^{2}-2 Y_{s}\left(Y_{s+}-Y_{s}\right)$.

\section{RBSDEs whose obstacles are not càdlàg in a general filtration.}

Let $T>0$ be a fixed terminal time. Let $f$ be a driver. Let $\xi=\left(\xi_{t}\right)_{t \in[0, T]}$ be a left-limited process in $\mathbb{S}^{2}$. We suppose moreover that the process $\xi$ is right uppersemicontinuous (r.u.s.c. for short). A process $\xi$ satisfying the previous properties will be called a barrier, or an obstacle.

Definition 3.1. A process $(Y, Z, \psi, M, A, C)$ is said to be a solution to the reflected BSDE with parameters $(f, \xi)$, where $f$ is a driver and $\xi$ is an obstacle, if $(Y, Z, \psi, M, A, C) \in \mathcal{E}^{2}(0, T)$ and

$$
\begin{array}{r}
Y_{\tau}=\xi_{T}+\int_{\tau}^{T} f\left(s, Y_{s}, Z_{s}, \psi_{s}\right) d s-\int_{\tau}^{T} Z_{s} d W_{s}-\int_{\tau}^{T} \int_{\mathcal{U}} \psi_{s}(u) \widetilde{\pi}(d u, d s)-\int_{\tau}^{T} d M_{s} \\
+A_{T}-A_{\tau}+C_{T-}-C_{\tau-} \text { for all } \tau \in \mathcal{T}_{0, T} \quad(3.1)
\end{array}
$$

$Y \geq \xi \quad($ up to an evanescent set) a.s.

$$
M \in \mathcal{M}_{l o c} \text { and } M_{0}=0 .
$$


In the above, the process $A$ is a nondecreasing right-continuous predictable process with $A_{0}=0, E\left(A_{T}\right)<\infty$ such that:

$$
\int_{0}^{T} 1_{\left\{Y_{t}>\xi_{t}\right\}} d A_{t}^{c}=0 \text { a.s. and }\left(Y_{\tau-}-\xi_{\tau-}\right)\left(A_{\tau}^{d}-A_{\tau-}^{d}\right)=0
$$

a.s. $\forall$ (predictable) $\tau \in \mathcal{T}_{0, T}$ and the process $C$ is a nondecreasing right-continuous adapted purely discontinuous process with $C_{0-}=0, E\left(C_{T}\right)<\infty$ such that:

$$
\left(Y_{\tau}-\xi_{\tau}\right)\left(C_{\tau}-C_{\tau-}\right)=0 \text { a.s. } \forall \tau \in \mathcal{T}_{0, T}
$$

Here $A^{c}$ denotes the continuous part of the nondecreasing process $A$ and $A^{d}$ its discontinuous part.

Remark 3.2. We note that a process $(Y, Z, \psi, M, A, C) \in \mathcal{E}^{2}(0, T)$ satisfies equation (3.1) in the above definition if and only if $\forall t \in[0, T]$, a.s.

$$
\begin{aligned}
Y_{t}= & \xi_{T}+\int_{t}^{T} f\left(s, Y_{s}, Z_{s}, \psi_{s}\right) d s-\int_{t}^{T} Z_{s} d W_{s}-\int_{t}^{T} \int_{\mathcal{U}} \psi_{s}(u) \widetilde{\pi}(d u, d s) \\
& -\int_{\tau}^{T} d M_{s}+A_{T}-A_{t}+C_{T-}-C_{t-} .
\end{aligned}
$$

Remark 3.3. If $(Y, Z, \psi, M, A, C) \in \mathcal{E}^{2}(0, T)$ satisfies the above definition, then the process $Y$ has left and right limits. Moreover, the process given by $\left(Y_{t}+\right.$ $\left.\int_{0}^{t} f\left(s, Y_{s}, Z_{s}, \psi_{s}\right) d s\right)_{t \in[0, T]}$ is a strong supermartingale.

The proof of the existence and uniqueness of the reflected BSDE solution defined above is based on a useful result (following lemma) in the case of $f$ depends only on $s$ and $\omega$ (i.e. $f(s, y, z, \psi)=f(s, \omega)$ ), the corollary 2.6 and the lemma 2.1. To this purpose, we first prove a lemma which will be used in the sequel.

Lemma 3.4. Let $\left(Y^{1}, Z^{1}, \psi^{1}, M^{1}, A^{1}, C^{1}\right) \in \mathcal{E}^{2}(0, T) \quad\left(\right.$ resp. $\quad\left(Y^{2}, Z^{2}, \psi^{2}, M^{2}, A^{1}\right.$, $\left.C^{2}\right) \in \mathcal{E}^{2}(0, T)$.) be a solution to the $R B S D E$ associated with driver $f^{1}(s, \omega)$ (resp. $\left.f^{2}(s, \omega)\right)$ and with obstacle $\xi$. There exists $c>0$ such that for all $\epsilon>0$, for all $\beta>\frac{1}{\epsilon^{2}}$ we have

$$
\left\|Z^{1}-Z^{2}\right\|_{\beta}^{2}+\left\|M^{1}-M^{2}\right\|_{\mathbb{M}_{\beta}^{2}}^{2}+\left\|\psi^{1}-\psi^{2}\right\|_{\mathbb{L}_{\pi}^{2, \beta}}^{2} \leq \epsilon^{2}\left\|f^{1}-f^{2}\right\|_{\beta}^{2}
$$

and

$$
\left\|\left|Y^{1}-Y^{2}\right|\right\|_{\beta}^{2} \leq 4 \epsilon^{2}\left(1+4 c^{2}\right)\left\|f^{1}-f^{2}\right\|_{\beta}^{2} .
$$

Proof: Let $\beta>0$ and $\epsilon>0$ be such that $\beta \geq \frac{1}{\epsilon^{2}}$. We set $\widetilde{Y}:=Y^{1}-Y^{2}, \widetilde{Z}:=$ $Z^{1}-Z^{2}, \widetilde{\psi}:=\psi^{1}-\psi^{2}, \widetilde{M}:=M^{1}-M^{2}, \widetilde{A}:=A^{1}-A^{2}, \widetilde{C}:=C^{1}-C^{2}$ and $\widetilde{f}(\omega, t):=f^{1}(\omega, t)-f^{2}(\omega, t)$. We note that $\widetilde{Y}_{T}:=\xi_{T}-\xi_{T}=0$. Moreover,

$$
\begin{array}{r}
\widetilde{Y}_{\tau}=\int_{\tau}^{T} \widetilde{f}(s) d s-\int_{\tau}^{T} \widetilde{Z}_{s} d W_{s}-\int_{\tau}^{T} \int_{\mathcal{U}} \widetilde{\psi}_{s}(u) \widetilde{\pi}(d u, d s)-\widetilde{M}_{T}+\widetilde{M}_{\tau}+\widetilde{A}_{T}-\widetilde{A}_{\tau} \\
+\widetilde{C}_{T-}-\widetilde{C}_{\tau-} \text { a.s. } \forall \tau \in \mathcal{T}_{0, T}, \quad \text { (3.8 }
\end{array}
$$

i.e.

$$
\widetilde{Y}_{\tau}=\widetilde{Y}_{0}-\int_{0}^{\tau} \widetilde{f}(s) d s+\int_{0}^{\tau} \widetilde{Z}_{s} d W_{s}+\int_{0}^{\tau} \int_{\mathcal{U}} \widetilde{\psi}_{s}(u) \widetilde{\pi}(d u, d s)+\widetilde{M}_{\tau}-\widetilde{A}_{\tau}-\widetilde{C}_{\tau-}
$$

a.s. $\forall \tau \in \mathcal{T}_{0, T}$, since $\widetilde{M}_{0}=M_{0}^{1}-M_{0}^{2}=0, \widetilde{A}_{0}=A_{0}^{1}-A_{0}^{2}=0$ and $\widetilde{C}_{0-}=$ $C_{0-}^{1}-C_{0-}^{2}=0$. Thus we see that $\tilde{Y}$ is an optional (strong) semimartingale with 
decomposition $\widetilde{Y}_{t}=\widetilde{Y}_{0}+N_{t}+A_{t}+B_{t}$, where $N_{t}=\int_{0}^{t} \widetilde{Z}_{s} d W_{s}+\int_{0}^{t} \int_{\mathcal{U}} \widetilde{\psi}_{s}(u) \widetilde{\pi}(d u, d s)+$ $\widetilde{M}_{t}, A_{t}=-\int_{0}^{t} \widetilde{f}(s) d s-\widetilde{A}_{t}$ and $B_{t}=-\widetilde{C}_{t-}$ (the notation is that of (2.5)). Applying Corollary 2.6 to $\widetilde{Y}$ gives: almost surely, for all $t \in[0, T]$,

$$
\begin{aligned}
e^{\beta t} \widetilde{Y}_{t}^{2} & =-\int_{0}^{t} \beta e^{\beta s} \widetilde{Y}_{s}^{2} d s+2 \int_{0}^{t} e^{\beta s} \widetilde{Y}_{s-} d(A+N)_{s} \\
& -\int_{0}^{t} e^{\beta s} d<N^{c}, N^{c}>_{s} \\
& -\sum_{0<s \leq t} e^{\beta s}\left(\widetilde{Y}_{s}-\widetilde{Y}_{s-}\right)^{2}-\int_{0}^{t} 2 e^{\beta s} \widetilde{Y}_{s} d(B)_{s+}-\sum_{0 \leq s<t} e^{\beta s}\left(\widetilde{Y}_{s+}-\widetilde{Y}_{s}\right)^{2} .
\end{aligned}
$$

Using the expressions of $N, A$ and $B$ and the fact that $\widetilde{Y}_{T}=0$, we get: almost surely, for all $t \in[0, T]$,

$$
\begin{aligned}
e^{\beta t} \widetilde{Y}_{t}^{2}+\int_{t}^{T} e^{\beta s} d<N^{c}, N^{c}>_{s} & =-\int_{t}^{T} \beta e^{\beta s} \widetilde{Y}_{s}^{2} d s+2 \int_{t}^{T} e^{\beta s} \widetilde{Y}_{s-} \widetilde{f}(s) d s \\
& +2 \int_{t}^{T} e^{\beta s} \widetilde{Y}_{s-} d \widetilde{A}-2 \int_{t}^{T} e^{\beta s} \widetilde{Y}_{s-} \widetilde{Z}_{s} d W_{s} \\
& -2 \int_{t}^{T} e^{\beta s} \widetilde{Y}_{s-} \int_{\mathcal{U}} \widetilde{\psi}_{s}(u) \widetilde{\pi}(d u, d s)-2 \int_{t}^{T} e^{\beta s} \widetilde{Y}_{s-} d \widetilde{M}_{s} \\
& -\sum_{t<s \leq T} e^{\beta s}\left(\widetilde{Y}_{s}-\widetilde{Y}_{s-}\right)^{2}+\int_{t}^{T} 2 e^{\beta s} \widetilde{Y}_{s} d(\widetilde{C})_{s} \\
& -\sum_{t \leq s<T} e^{\beta s}\left(\widetilde{Y}_{s+}-\widetilde{Y}_{s}\right)^{2} .
\end{aligned}
$$

Then

$$
\begin{aligned}
e^{\beta t} \widetilde{Y}_{t}^{2}+\int_{t}^{T} e^{\beta s} \widetilde{Z}_{s}^{2} d s & +\int_{t}^{T} e^{\beta s} \int_{\mathcal{U}}\left|\widetilde{\psi}_{s}(u)\right|^{2} \mu(d u) d s+\int_{t}^{T} e^{\beta s} d<\widetilde{M}^{c}, \widetilde{M}^{c}>_{s}= \\
& -\int_{t}^{T} \beta e^{\beta s} \widetilde{Y}_{s}^{2} d s+2 \int_{t}^{T} e^{\beta s} \widetilde{Y}_{s-} \widetilde{f}(s) d s \\
& +2 \int_{t}^{T} e^{\beta s} \widetilde{Y}_{s-} d \widetilde{A}-2 \int_{t}^{T} e^{\beta s} \widetilde{Y}_{s-} \widetilde{Z}_{s} d W_{s} \\
& -2 \int_{t}^{T} e^{\beta s} \widetilde{Y}_{s-} \int_{\mathcal{U}} \widetilde{\psi}_{s}(u) \widetilde{\pi}(d u, d s)-2 \int_{t}^{T} e^{\beta s} \widetilde{Y}_{s-} d \widetilde{M}_{s} \\
& -\sum_{t<s \leq T} e^{\beta s}\left(\widetilde{Y}_{s}-\widetilde{Y}_{s-}\right)^{2}+\int_{t}^{T} 2 e^{\beta s} \widetilde{Y}_{s} d(\widetilde{C})_{s} \\
& -\sum_{t \leq s<T} e^{\beta s}\left(\widetilde{Y}_{s+}-\widetilde{Y}_{s}\right)^{2} .
\end{aligned}
$$

It is clear that for all $t \in[0, T]-\sum_{t<s<T} e^{\beta s}\left(\widetilde{Y}_{s}-\widetilde{Y}_{s-}\right)^{2}-\sum_{t<s<T} e^{\beta s}\left(\widetilde{Y}_{s+}-\widetilde{Y}_{s}\right)^{2} \leq$ 0 . By applying the inequality $2 a b \leq\left(\frac{a}{\epsilon}\right)^{2}+\epsilon^{2} b^{2}$, valid for all $(a, b)$ in $\mathbb{R}^{2}$, we get: 
a.e. for all $t \in[0, T]$

$$
\begin{aligned}
-\int_{t}^{T} \beta e^{\beta s} \widetilde{Y}_{s}^{2} d s+2 \int_{t}^{T} e^{\beta s} \widetilde{Y}_{s-} \widetilde{f}(s) d s & \leq-\int_{t}^{T} \beta e^{\beta s} \widetilde{Y}_{s}^{2} d s+\frac{1}{\epsilon^{2}} \int_{t}^{T} e^{\beta s} \widetilde{Y}_{s-}^{2} d s \\
& +\epsilon^{2} \int_{t}^{T} e^{\beta s} \widetilde{f}(s)^{2} d s \\
& =\left(\frac{1}{\epsilon^{2}}-\beta\right) \int_{t}^{T} e^{\beta s} \widetilde{Y}_{s-}^{2} d s+\epsilon^{2} \int_{t}^{T} e^{\beta s} \tilde{f}(s)^{2} d s .
\end{aligned}
$$

As $\beta \geq \frac{1}{\epsilon^{2}}$, we have $\left(\frac{1}{\epsilon^{2}}-\beta\right) \int_{t}^{T} e^{\beta s} \widetilde{Y}_{s-}^{2} d s \leq 0$ for all $t \in[0, T]$ a.s.

Next, we have also that the term $\int_{t}^{T} e^{\beta s} \widetilde{Y}_{s} d \widetilde{C}_{s}$ is non-positive. Indeed a.s. for all $t \in[0, T]$,

$$
\int_{t}^{T} e^{\beta s} \widetilde{Y}_{s} d \widetilde{C}_{s}=\sum_{t \leq s<T} e^{\beta s} \widetilde{Y}_{s} \triangle \widetilde{C}_{s}
$$

and a.s. for all $t \in[0, T]$

$$
\widetilde{Y}_{t} \triangle \widetilde{C}_{t}=\left(Y_{t}^{1}-Y_{t}^{2}\right) \triangle C_{t}^{1}-\left(Y_{t}^{1}-Y_{t}^{2}\right) \triangle C_{t}^{2} .
$$

We use property (3.5) of $C^{1}$ and the fact that $Y^{2} \geq \xi$ to obtain: a.s. for all $t \in[0, T]$

$$
\left(Y_{t}^{1}-Y_{t}^{2}\right) \triangle C_{t}^{1}=\left(Y_{t}^{1}-\xi_{t}\right) \triangle C_{t}^{1}-\left(Y_{t}^{2}-\xi_{t}\right) \triangle C_{t}^{1}=0-\left(Y_{t}^{2}-\xi_{t}\right) \triangle C_{t}^{1} \leq 0
$$

Similarly, we obtain: a.s. for all $t \in[0, T]$,

$$
\left(Y_{t}^{1}-Y_{t}^{2}\right) \triangle C_{t}^{2}=\left(Y_{t}^{1}-\xi_{t}\right) \triangle C_{t}^{2}-\left(Y_{t}^{2}-\xi_{t}\right) \triangle C_{t}^{2}=\left(Y_{t}^{1}-\xi_{t}\right) \triangle C_{t}^{2}-0 \geq 0 .
$$

We also show that $\int_{t}^{T} e^{\beta s} \widetilde{Y}_{s-} d \widetilde{A}$ is non-positive by using property (3.4) of the definition of the RBSDE and the fact that $Y^{i} \geq \xi$ for $i=1,2$ and that $A^{i}=$ $A^{i, c}+A^{i, d}$ (see also Quenez and Sulem, 2014). Then

$$
\begin{gathered}
e^{\beta t} \widetilde{Y}_{t}^{2}+\int_{t}^{T} e^{\beta s} \widetilde{Z}_{s}^{2} d s+\int_{t}^{T} e^{\beta s} d<\widetilde{M}^{c}, \widetilde{M}^{c}>_{s}+\int_{t}^{T} e^{\beta s} \int_{\mathcal{U}}\left|\widetilde{\psi}_{s}(u)\right|^{2} \mu(d u) d s \leq \\
\epsilon^{2} \int_{t}^{T} e^{\beta s} \widetilde{f}^{2}(s) d s-2 \int_{t}^{T} e^{\beta s} \widetilde{Y}_{s-} \widetilde{Z}_{s} d W_{s} \\
-2 \int_{t}^{T} \int_{\mathcal{U}} e^{\beta s} \widetilde{Y}_{s-} \widetilde{\psi}_{s}(u) \widetilde{\pi}(d u, d s)-2 \int_{t}^{T} e^{\beta s} \widetilde{Y}_{s-} d \widetilde{M}_{s} \quad \forall \text { a.s. } t \in[0, T] . \quad(3.10
\end{gathered}
$$

We now show that the term $\int_{0}^{T} e^{\beta s} \widetilde{Y}_{s-} \widetilde{Z}_{s} d W_{s}$ has zero expectation. To this purpose, we show that $E\left[\sqrt{\int_{0}^{T} e^{2 \beta s} \widetilde{Y}_{s-}^{2} \widetilde{Z}_{s}^{2} d s}\right]<\infty$, in the same way that in the proof of Lemma 3.2 (A priori estimates) in Grigorova et al. (2015). By using the leftcontinuity of a.e. trajectory of the process $\left(\widetilde{Y}_{s-}\right)$, we have

$$
\left(\widetilde{Y}_{s-}\right)^{2}(\omega) \leq \sup _{t \in \mathbb{Q}}\left(\widetilde{Y}_{t-}\right)^{2}(\omega) \text { for all } s \in(0, T], \text { for a.s. } \omega \in \Omega .
$$

On the other hand, for all $t \in(0, T]$, a.s., $\left(\widetilde{Y}_{t-}\right)^{2} \leq e s s \sup _{\tau \in \mathcal{T}_{0, T}}\left(\widetilde{Y}_{\tau}\right)^{2}$. Then

$$
\sup _{t \in \mathbb{Q}}\left(\widetilde{Y}_{t-}\right)^{2} \leq e s s \sup _{\tau \in \mathcal{T}_{0, T}}\left(\widetilde{Y}_{\tau}\right)^{2} \quad \text { a.s. }
$$


According to (3.11) and (3.12) we obtain

$$
\int_{0}^{T} e^{2 \beta s} \widetilde{Y}_{s-}^{2} \widetilde{Z}_{s}^{2} d s \leq \int_{0}^{T} e^{2 \beta s} \sup _{t \in \mathbb{Q}} \widetilde{Y}_{t-}^{2} \widetilde{Z}_{s}^{2} d s \leq \int_{0}^{T} e^{2 \beta s} \text { ess } \sup _{\tau \in \mathcal{T}_{0, T}} \widetilde{Y}_{\tau}^{2} \widetilde{Z}_{s}^{2} d s .
$$

Using (3.13), together with Cauchy-Schwarz inequality, gives

$$
E\left[\sqrt{\int_{0}^{T} e^{2 \beta s} \widetilde{Y}_{s-}^{2} \widetilde{Z}_{s}^{2} d s}\right] \leq E\left[\sqrt{e s s \sup _{\tau \in \mathcal{T}_{0, T}} \widetilde{Y}_{\tau}^{2}} \sqrt{\int_{0}^{T} e^{2 \beta s} \widetilde{Z}_{s}^{2} d s}\right]
$$

Then

$$
E\left[\sqrt{\int_{0}^{T} e^{2 \beta s} \widetilde{Y}_{s-}^{2} \widetilde{Z}_{s}^{2} d s}\right] \leq\|\widetilde{Y}\|_{\mathbb{S}^{2}}\|\widetilde{Z}\|_{2 \beta} .
$$

We conclude that $E\left[\sqrt{\int_{0}^{T} e^{2 \beta s} \widetilde{Y}_{s-}^{2} \widetilde{Z}_{s}^{2} d s}\right]<\infty$, whence, $E\left[\int_{0}^{T} e^{\beta s} \widetilde{Y}_{s-} \widetilde{Z}_{s} d W_{s}\right]=0$. Next we show that $E\left[\int_{0}^{T} \int_{\mathcal{U}} e^{\beta s} \widetilde{Y}_{s-} \widetilde{\psi}_{s}(u) \widetilde{\pi}(d u, d s)\right]=0$. For this purpose, we first prove that $E\left[\sqrt{\int_{0}^{T} \int_{\mathcal{U}} e^{2 \beta s} \widetilde{Y}_{s-}^{2} \widetilde{\psi}_{s}^{2}(u) \mu(d u) d s}\right]<\infty$. According to (3.11) and (3.12), we have

$$
\begin{aligned}
\int_{0}^{T} \int_{\mathcal{U}} e^{2 \beta s} \widetilde{Y}_{s-}^{2} \widetilde{\psi}_{s}^{2}(u) \mu(d u) d s & \leq \int_{0}^{T} \int_{\mathcal{U}} e^{2 \beta s} \sup _{t \in \mathbb{Q}} \widetilde{Y}_{t-}^{2} \widetilde{\psi}_{s}^{2}(u) \mu(d u) d s \\
& \leq \int_{0}^{T} \int_{\mathcal{U}} e^{2 \beta s} \text { ess } \sup _{\tau \in \mathcal{T}_{0}, T} \widetilde{Y}_{\tau}^{2} \widetilde{\psi}_{s}^{2}(u) \mu(d u) d s .
\end{aligned}
$$

Using (3.15) and Cauchy-Schwarz inequality, gives

$$
E\left[\sqrt{\int_{0}^{T} \int_{\mathcal{U}} e^{2 \beta s} \widetilde{Y}_{s-}^{2} \widetilde{\psi}_{s}^{2}(u) \mu(d u) d s}\right] \leq E\left[\sqrt{e s s \sup _{\tau \in \mathcal{T}_{0}, T} \widetilde{Y}_{\tau}^{2}} \sqrt{\int_{0}^{T} e^{2 \beta s} \int_{\mathcal{U}} \widetilde{\psi}_{s}^{2}(u) \mu(d u) d s}\right] .
$$

Thus

$$
E\left[\sqrt{\left.\int_{0}^{T} \int_{\mathcal{U}} e^{2 \beta s} \widetilde{Y}_{s-}^{2} \widetilde{\psi}_{s}^{2}(u) \mu(d u) d s\right]} \leq\|\widetilde{Y}\|_{\mathbb{S}^{2}}\|\widetilde{\psi}\|_{\mathbb{L}_{\pi}^{2,2 \beta}}<\infty .\right.
$$

Then $E\left[\int_{0}^{T} \int_{\mathcal{U}} e^{\beta s} \widetilde{Y}_{s-} \widetilde{\psi}_{s}(u) \widetilde{\pi}(d u, d s)\right]=0$. Finally the same result holds for the martingale $\int_{0}^{t} e^{\beta s} \widetilde{Y}_{s-} d \widetilde{M}_{s}$, since:

$$
E\left[\sqrt{\int_{0}^{T} e^{2 \beta s} \widetilde{Y}_{s-}^{2} d[\widetilde{M}]_{s}}\right] \leq\|\widetilde{Y}\|_{S^{2}}\|\widetilde{M}\|_{\mathbb{M}_{2 \beta}^{2}}<\infty .
$$

By taking expectations on both sides of (3.10) with $t=0$, we obtain:

$$
\begin{aligned}
& \widetilde{Y}_{0}^{2}+E\left[\int_{0}^{T} e^{\beta s} \widetilde{Z}_{s}^{2} d s\right]+E\left[\int_{0}^{T} e^{\beta s} d<\widetilde{M}>_{s}\right]+E\left[\int_{0}^{T} e^{\beta s} \int_{\mathcal{U}}\left|\widetilde{\psi}_{s}(u)\right|^{2} \mu(d u) d s\right] \\
& \leq \epsilon^{2}\|\widetilde{f}(s)\|_{\beta}^{2} .
\end{aligned}
$$

Hence, with the fact that $E\left[\int_{0}^{T} e^{\beta s} d<\widetilde{M}>_{s}\right]=E\left[\int_{0}^{T} e^{\beta s} d[\widetilde{M}]_{s}\right]$, we have

$$
\|\widetilde{Z}\|_{\beta}^{2}+\|\widetilde{M}\|_{\mathbb{M}_{\beta}^{2}}^{2}+\|\widetilde{\psi}\|_{\mathbb{L}_{\pi}^{2, \beta}}^{2} \leq \epsilon^{2}\|\widetilde{f}(s)\|_{\beta}^{2}
$$


This therefore shows the first inequality of the lemma. From (3.10) we also get, for all $\tau \in \mathcal{T}_{0, T}$

$$
\begin{array}{r}
e^{\beta \tau} \widetilde{Y}_{\tau}^{2} \leq \epsilon^{2} \int_{0}^{T} e^{\beta s} \widetilde{f}^{2}(s) d s-2 \int_{\tau}^{T} e^{\beta s} \widetilde{Y}_{s-} \widetilde{Z}_{s} d W_{s}-2 \int_{\tau}^{T} \int_{\mathcal{U}} e^{\beta s} \widetilde{Y}_{s-} \widetilde{\psi}_{s}(u) \widetilde{\pi}(d u, d s) \\
-2 \int_{\tau}^{T} e^{\beta s} \widetilde{Y}_{s-} d \widetilde{M}_{s} \quad \text { a.s. }
\end{array}
$$

By taking first the essential supremum over $\tau \in \mathcal{T}_{0, T}$, and then the expectation on both sides of the inequality (3.19), we obtain:

$$
\begin{array}{r}
E\left[\text { ess } \sup _{\tau \in \mathcal{T}_{0, T}} e^{\beta \tau} \widetilde{Y}_{\tau}^{2}\right] \leq \epsilon^{2}\|\widetilde{f}(s)\|_{\beta}^{2}+2 E\left[\text { ess } \sup _{\tau \in \mathcal{T}_{0, T}}\left|\int_{0}^{\tau} e^{\beta s} \widetilde{Y}_{s-} \widetilde{Z}_{s} d W_{s}\right|\right] \\
+2 E\left[\text { ess } \sup _{\tau \in \mathcal{T}_{0, T}}\left|\int_{0}^{\tau} \int_{\mathcal{U}} e^{\beta s} \widetilde{Y}_{s-} \widetilde{\psi}_{s}(u) \widetilde{\pi}(d u, d s)\right|\right] \\
+2 E\left[e s s \sup _{\tau \in \mathcal{T}_{0, T}}\left|\int_{0}^{\tau} e^{\beta s} \widetilde{Y}_{s-} d \widetilde{M}_{s}\right|\right] .
\end{array}
$$

By using the continuity of a.e. trajectory of the process $\left(\int_{0}^{t} e^{\beta s} \widetilde{Y}_{s}-\widetilde{Z}_{s} d W_{s}\right)_{t \in[0, T]}$ (Grigorova et al., 2015, Prop. A.3) and Burkholder-Davis-Gundy inequalities (Protter, 1990, Theorem 48, page 193. Applied with $p=1$ ), we get

$$
\begin{array}{r}
E\left[e s s \sup _{\tau \in \mathcal{T}_{0, T}}\left|\int_{0}^{\tau} e^{\beta s} \widetilde{Y}_{s-} \widetilde{Z}_{s} d W_{s}\right|\right]=E\left[\sup _{t \in[0, T]}\left|\int_{0}^{t} e^{\beta s} \widetilde{Y}_{s-} \widetilde{Z}_{s} d W_{s}\right|\right] \\
\leq c E\left[\sqrt{\int_{0}^{T} e^{2 \beta s} \widetilde{Y}_{s-}^{2} \widetilde{Z}_{s}^{2} d s}\right]
\end{array}
$$

where $c$ is a positive "universal" constant (which does not depend on the other parameters). The same reasoning as that used to obtain equation (3.13) leads to

$$
\sqrt{\int_{0}^{T} e^{2 \beta s} \widetilde{Y}_{s-}^{2} \widetilde{Z}_{s}^{2} d s} \leq \sqrt{e s s \sup _{\tau \in \mathcal{T}_{0, T}} e^{\beta \tau} \widetilde{Y}_{\tau}^{2} \int_{0}^{T} e^{\beta s} \widetilde{Z}_{s}^{2} d s} \quad \text { p.s. }
$$

From the inequalities (3.21), (3.22) and $a b \leq \frac{1}{4} a^{2}+b^{2}$, we have

$$
2 E\left[e s s \sup _{\tau \in \mathcal{T}_{0, T}}\left|\int_{0}^{\tau} e^{\beta s} \widetilde{Y}_{s-} \widetilde{Z}_{s} d s\right|\right] \leq \frac{1}{4} E\left[e s s \sup _{\tau \in \mathcal{T}_{0, T}} e^{\beta \tau} \widetilde{Y}_{\tau}^{2}\right]+4 c^{2} E\left[\int_{0}^{T} e^{\beta s} \widetilde{Z}_{s}^{2} d s\right] .
$$

By the same arguments, we have

$$
\begin{aligned}
2 E\left[e s s \sup _{\tau \in \mathcal{T}_{0, T}}\left|\int_{0}^{\tau} e^{\beta s} \widetilde{Y}_{s-} \widetilde{\psi}_{s}(u) \widetilde{\pi}(d u, d s)\right|\right] \leq 2 c E\left[\sqrt{\left|\int_{0}^{T} \int_{\mathcal{U}} e^{2 \beta s} \widetilde{Y}_{s-}^{2} \widetilde{\psi}_{s}^{2}(u) \mu(d u) d s\right|}\right] \\
\leq \frac{1}{4} E\left[e s s \sup _{\tau \in \mathcal{T}_{0, T}} e^{\beta \tau} \widetilde{Y}_{\tau}^{2}\right]+4 c^{2} E\left[\int_{0}^{T} \int_{\mathcal{U}} e^{\beta s} \widetilde{\psi}_{s}^{2}(u) \mu(d u) d s\right]
\end{aligned}
$$


and

$$
\begin{aligned}
2 E\left[e s s \sup _{\tau \in \mathcal{T}_{0, T}}\left|\int_{0}^{\tau} e^{\beta s} \widetilde{Y}_{s-} d \widetilde{M}_{s}\right|\right] \leq 2 c E\left[\sqrt{\left|\int_{0}^{T} e^{2 \beta s} \widetilde{Y}_{s-}^{2} d[\widetilde{M}]_{s}\right|}\right] \\
\leq \frac{1}{4} E\left[e s s \sup _{\tau \in \mathcal{T}_{0, T}} e^{\beta \tau} \widetilde{Y}_{\tau}^{2}\right]+4 c^{2} E\left[\int_{0}^{T} e^{\beta s} d[\widetilde{M}]_{s}\right]
\end{aligned}
$$

where $c$ is a positive constant which does not depend on the other parameters. From (3.21), (3.23), (3.24) and (3.25), we get

$$
\frac{1}{4}\||\widetilde{Y}|\|_{\beta}^{2} \leq \epsilon^{2}\|\widetilde{f}(s)\|_{\beta}^{2}+4 c^{2}\|\widetilde{Z}\|_{\beta}^{2}+4 c^{2}\|\widetilde{M}\|_{\mathbb{M}_{\beta}^{2}}^{2}+4 c^{2}\|\widetilde{\psi}\|_{\mathbb{L}_{\pi}^{2, \beta}}^{2} .
$$

This inequality, combined with (3.18), gives

$$
\||\widetilde{Y}|\|_{\beta}^{2} \leq 4 \epsilon^{2}\left(1+4 c^{2}\right)\|\widetilde{f}(s)\|_{\beta}^{2} .
$$

In the following lemma, we prove existence and uniqueness of the solution to the RBSDE from Definition 3.1 in the case where the driver $f$ depends only on $s$ and $\omega$, i.e. $f(\omega, s, y, z, \psi):=f(\omega, s)$.

Lemma 3.5. Suppose that $f$ does not depend on $y, z, \psi$ that is $f(\omega, s, y, z, \psi):=$ $f(\omega, s)$, where $f$ is a process in $\mathbb{H}^{2}$. Let $\xi$ be an obstacle. Then, the RBSDE from Definition 3.1 admits a unique solution $(Y, Z, \psi, M, A, C) \in \mathcal{E}^{2}(0, T)$, and for each $S \in \mathcal{T}_{0, T}$, we have

$$
Y_{S}=e s s \sup _{\tau \in \mathcal{T}_{S, T}} E\left[\xi_{\tau}+\int_{S}^{\tau} f(t) d t \mid \mathcal{F}_{S}\right] \text { a.s. }
$$

Proof: For all $S \in \mathcal{T}_{0, T}$, we define $\bar{Y}(S)$ by:

$$
\bar{Y}(S)=e s s \sup _{\tau \in \mathcal{T}_{S, T}} E\left[\xi_{\tau}+\int_{S}^{\tau} f(t) d t \mid \mathcal{F}_{S}\right], \quad \bar{Y}(T)=\xi_{T} .
$$

And $\overline{\bar{Y}}(S)$ by:

$$
\overline{\bar{Y}}(S)=\bar{Y}(S)+\int_{0}^{S} f(t) d t=e s s \sup _{\tau \in \mathcal{T}_{S, T}} E\left[\xi_{\tau}+\int_{0}^{\tau} f(t) d t \mid \mathcal{F}_{S}\right] .
$$

We note that the process $\left(\xi_{t}+\int_{0}^{t} f(s) d s\right)_{t \in[0, T]}$ is progressive. Therefore, the family $(\overline{\bar{Y}}(S))_{S \in \mathcal{T}_{0, T}}$ is a supermartingale family (see Kobylanski and Quenez, 2012, Remark 1.2 with Prop.1.5), and with remark (b) in (Dellacherie and Meyer, 1980, page 435), gives the existence of a strong optional supermartingale (which we denote again by $\overline{\bar{Y}})$ such that $\overline{\bar{Y}}_{S}=\overline{\bar{Y}}(S)$ a.s. for all $S \in \mathcal{T}_{0, T}$. Thus, we have $\bar{Y}(S)=\overline{\bar{Y}}(S)-\int_{0}^{S} f(t) d t=\overline{\bar{Y}}_{S}-\int_{0}^{S} f(t) d t$ a.s. for all $S \in \mathcal{T}_{0, T}$ (see Dellacherie and Meyer, 1980). On the other hand, we know that almost all trajectories of the strong optional supermartingale $\overline{\bar{Y}}$ are làdlàg. Thus, we get that the làdlàg optional process $\left(\bar{Y}_{t}\right)_{t \in[0, T]}=\left(\overline{\bar{Y}}_{t}-\int_{0}^{t} f(s) d s\right)_{t \in[0, T]}$ aggregates the family $(\bar{Y}(S))_{S \mathcal{T}_{0, T}}$.

To prove the lemma 3.5 , it must be shown, as a first step, that $\bar{Y} \in \mathbb{S}^{2}$ by giving an estimate of $\||\bar{Y}|\|_{\mathbb{S}^{2}}^{2}$ in terms of $\|\mid \xi\|_{\mathbb{S}^{2}}^{2}$ and $\|f\|_{\mathbb{H}^{2}}^{2}$. In the second step, we exhibit processes $Z, \psi, M, A$ and $C$ such that $(\bar{Y}, Z, \psi, M, A, C)$ is a solution to the $\operatorname{RBSDE}$ with parameters $(f, \xi)$. In the third step, we prove that $A \times C \in \mathbb{S}^{2} \times \mathbb{S}^{2}$ 
and we give an estimate of $\||A|\|_{\mathbb{S}^{2}}^{2}$ and $\||C|\|_{\mathbb{S}^{2}}^{2}$. In the fourth step, we show that $Z \in \mathbb{H}^{2}, \psi \in \mathbb{L}_{\pi}^{2}$ and $M \in \mathbb{M}^{2}$, and finally we show the uniqueness of the solution. Step 1. By using the definition of $\bar{Y}$ (3.27), Jensen's inequality and the triangular inequality, we get

$$
\left|\bar{Y}_{S}\right| \leq e s s \sup _{\tau \in \mathcal{T}_{S, T}} E\left[\left|\xi_{\tau}\right|+\left|\int_{S}^{\tau} f(t) d t\right| \mid \mathcal{F}_{S}\right] \leq E\left[\text { ess } \sup _{\tau \in \mathcal{T}_{S, T}}\left|\xi_{\tau}\right|+\int_{0}^{T}|f(t)| d t \mid \mathcal{F}_{S}\right]
$$

Thus, we obtain

with

$$
\left|\bar{Y}_{S}\right| \leq E\left[X \mid \mathcal{F}_{S}\right]
$$

$$
X=\int_{0}^{T}|f(t)| d t+e s s \sup _{\tau \in \mathcal{T}_{0, T}}\left|\xi_{\tau}\right|
$$

Applying Cauchy-Schwarz inequality gives

$$
E\left[X^{2}\right] \leq c T\|f\|_{\mathbb{H}^{2}}^{2}+c\||\xi|\|_{\mathbb{S}^{2}}^{2}<\infty .
$$

where $c$ is a positive constant. Now, inequality (3.29) leads to $\left|\bar{Y}_{S}\right|^{2} \leq\left|E\left[X \mid \mathcal{F}_{S}\right]\right|^{2}$. By taking the essential supremum over $S \in \mathcal{T}_{0, T}$ we get ess $\sup _{S \in \mathcal{T}_{0, T}}\left|\bar{Y}_{S}\right|^{2} \leq$ ess $\sup _{S \in \mathcal{T}_{0, T}}\left|E\left[X \mid \mathcal{F}_{S}\right]\right|^{2}$. By using Proposition A.3 in Grigorova et al. (2015), we get ess $\sup _{S \in \mathcal{T}_{0, T}}\left|\bar{Y}_{S}\right|^{2} \leq \sup _{t \in[0, T]}\left|E\left[X \mid \mathcal{F}_{t}\right]\right|^{2}$. By using this inequality and Doob's martingale inequalities, we obtain

$$
E\left[\text { ess } \sup _{S \in \mathcal{T}_{0, T}}\left|\bar{Y}_{S}\right|^{2}\right] \leq E\left[\sup _{t \in[0, T]}\left|E\left[X \mid \mathcal{F}_{t}\right]\right|^{2}\right] \leq c E\left[X^{2}\right]
$$

where $c$ is a positive constant that changes from line to line. Finally, combining inequalities (3.31) and (3.32) gives

$$
E\left[\text { ess } \sup _{S \in \mathcal{T}_{0, T}}\left|\bar{Y}_{S}\right|^{2}\right] \leq c T\|f\|_{\mathrm{H}^{2}}^{2}+c\||\xi|\|_{\mathbb{S}^{2}}^{2}<\infty
$$

Then $\bar{Y}_{S} \in \mathbb{S}^{2}$.

Step 2. Due to the previous step and to the assumption $f \in \mathbb{H}^{2}$, the strong optional supermartingale $\overline{\bar{Y}}$ is of class $(D)$. Applying Mertens decomposition (Grigorova et al., 2015, Theorem A.1) and a result from optimal stopping theory (see more in El Karoui, 1981, Prop. 2.34. page 131 or Kobylanski and Quenez, 2012), gives the following

$$
\begin{gathered}
\overline{\bar{Y}}_{\tau}=N_{\tau}-A_{\tau}-C_{\tau-} \forall \tau \in \mathcal{T}_{0, T} \\
\bar{Y}_{\tau}=-\int_{0}^{\tau} f(t) d t+N_{\tau}-A_{\tau}-C_{\tau-} \text { a.s. } \forall \tau \in \mathcal{T}_{0, T}
\end{gathered}
$$

where $N$ is a (càdlàg) uniformly integrable martingale such that $N_{0}=0, A$ is a nondecreasing right-continuous predictable process such that $A_{0}=0, E\left(A_{T}\right)<\infty$ and satisfying (3.4), and $C$ is a nondecreasing right-continuous adapted purely discontinuous process such that $C_{0-}=0, E\left(C_{T}\right)<\infty$ and satisfying (3.5). By the martingale representation theorem (Lemma 2.1), there exists a unique predictable process $Z$, a unique process $\psi$ and a unique (càdlàg) local martingales orthogonal $M$ such that

$$
N_{t}=\int_{0}^{t} Z_{s} d W_{s}+\int_{0}^{t} \int_{\mathcal{U}} \psi_{s}(u) \widetilde{\pi}(d u, d s)+M_{t}
$$

Moreover, we have $\bar{Y}_{T}=\xi_{T}$ a.s. by definition of $\bar{Y}$. Combining this with equation (3.34). gives equation (3.1). Also by definition of $\bar{Y}$, we have $\bar{Y}_{S} \geq \xi_{S}$ a.s. for 
all $S \in \mathcal{T}_{0, T}$, which, along with Proposition A.4 in Grigorova et al. (2015) (or Theorem 3.2. in Nikeghbali, 2006), shows that $\bar{Y}$ satisfies inequality (3.2). Finally, to conclude that the process $(\bar{Y}, Z, \psi, M, A, C)$ is a solution to the RBSDE with parameters $(f, \xi)$, it remains to show that $Z \times \psi \times M \times A \times C \in \mathbb{H}^{2} \times \mathbb{L}_{\pi}^{2} \times \mathbb{M}^{2} \times \mathbb{S}^{2} \times \mathbb{S}^{2}$. Step 3. Let us show that $A \times C \in \mathbb{S}^{2} \times \mathbb{S}^{2}$.

Let us define the process $\overline{\bar{A}}_{t}=A_{t}+C_{t-}$ where the processes $A$ and $C$ are given by (3.34). By arguments similar to those used in the proof of inequality (3.29), we see that $\left|\overline{\bar{Y}}_{S}\right| \leq E\left[X \mid \mathcal{F}_{S}\right]$ with

$$
X=\int_{0}^{T}|f(t)| d t+e s s \sup _{\tau \in \mathcal{T}_{S, T}}\left|\xi_{\tau}\right| .
$$

Then, the Corollary A.1 in Grigorova et al. (2015) ensures the existence of a constant $c>0$ such that $E\left[\left(\overline{\bar{A}}_{T}\right)^{2}\right] \leq c E\left[X^{2}\right]$. By combining this inequality with inequality (3.31), we obtain

$$
E\left[\left(\overline{\bar{A}}_{T}\right)^{2}\right] \leq c T\|f\|_{\mathbb{H}^{2}}^{2}+c\||\xi|\|_{\mathbb{S}^{2}}^{2}
$$

where we have again allowed the positive constant $c$ to vary from line to line. We conclude that $\overline{\bar{A}} \in L^{2}$. And with the nondecreasingness of $\overline{\bar{A}}$, then $\left(\overline{\bar{A}}_{\tau}\right)^{2} \leq\left(\overline{\bar{A}}_{T}\right)^{2}$ for all $\tau \in \mathcal{T}_{0, T}$ thus

$$
E\left[\text { ess } \sup _{\tau \in \mathcal{T}_{0}, T}\left(\overline{\bar{A}}_{\tau}\right)^{2}\right] \leq E\left[\left(\overline{\bar{A}}_{T}\right)^{2}\right]
$$

i.e. $\overline{\bar{A}} \in \mathbb{S}^{2}$ then $A \in \mathbb{S}^{2}$ and $C \in \mathbb{S}^{2}$.

Step 4. Let us now prove that $Z \times \psi \times M \in \mathbb{H}^{2} \times \mathbb{L}_{\pi}^{2} \times \mathbb{M}^{2}$. We have from step 3

$$
\int_{0}^{T} Z_{s} d W_{s}+\int_{0}^{T} \int_{\mathcal{U}} \psi_{s}(u) \widetilde{\pi}(d u, d s)+M_{T}=\bar{Y}_{T}+\int_{0}^{T} f(t) d t+\overline{\bar{A}}_{T}-\bar{Y}_{0}
$$

where $\overline{\bar{A}}$ is the process from Step 3 . Since $\overline{\bar{A}}_{T} \in L^{2}, \bar{Y}_{T} \in L^{2}, \bar{Y}_{0} \in L^{2}$ and $f \in \mathbb{H}^{2}$. Hence, $\int_{0}^{T} Z_{s} d W_{s} \in L^{2}, \int_{0}^{T} \int_{\mathcal{U}} \psi_{s}(u) \widetilde{\pi}(d u, d s) \in L^{2}$ and $M_{T} \in L^{2}$ and consequently $Z \times \psi \times M \in \mathbb{H}^{2} \times \mathbb{L}_{\pi}^{2} \times \mathbb{M}^{2}$.

For the uniqueness of the solution, suppose that $(Y, Z, \psi, M, A, C)$ is a solution of the RBSDE with driver $f$ and obstacle $\xi$. Then, by the previous inequality 3.7 in the Lemma 3.4 (applied with $f^{1}=f^{2}=f$ ) we obtain $Y=\bar{Y}$ in $\mathbb{S}^{2}$, where $\bar{Y}$ is given by (3.27). The uniqueness of $A, C, Z, \psi$ and $M$ follows from the uniqueness of Mertens decomposition of strong optional supermartingales and from the uniqueness of the martingale representation (Lemma 2.1).

Remark 3.6. (1) We note that the uniqueness of $Z, \psi$ and $M$ can be obtained also by applying (3.6) in the previous Lemma 3.4.

(2) Let $\beta>0$. For $\phi \in \mathbb{S}^{2}$, we have the inequality $E\left[\int_{0}^{T} e^{\beta t}\left|\phi_{t}\right|^{2} d t\right] \leq$ $T E\left[\right.$ ess $\left.\sup _{\tau \in \mathcal{T}_{0, T}} e^{\beta \tau}\left|\phi_{\tau}\right|^{2}\right]$. Indeed, by applying Fubini's theorem, we get

$$
\begin{aligned}
& E\left[\int_{0}^{T} e^{\beta t}\left|\phi_{t}\right|^{2} d t\right]=\int_{0}^{T} E\left[e^{\beta t}\left|\phi_{t}\right|^{2}\right] d t \leq \int_{0}^{T} E\left[e s s \sup _{\tau \in \mathcal{T}_{0, T}} e^{\beta \tau}\left|\phi_{\tau}\right|^{2}\right] d s= \\
& T E\left[e s s \sup _{\tau \in \mathcal{T}_{0, T}} e^{\beta \tau}\left|\phi_{\tau}\right|^{2}\right] .
\end{aligned}
$$


In the following theorem, we prove existence and uniqueness of the solution to the RBSDE from Definition 3.1 in the case of a general Lipschitz driver $f$ by using a fixed-point theorem and by using (2) in the Remark 3.6 .

Theorem 3.7. Let $\xi$ be a left-limited and r.u.s.c. process in $\mathbb{S}^{2}$ and let $f$ be a Lipschitz driver. The RBSDE with parameters $(f, \xi)$ from Definition 3.1 admits a unique solution $(Y, Z, \psi, M, A, C) \in \mathcal{E}^{2}(0, T)$.

Proof: We note by $\mathcal{E}_{f}^{\beta}$ the space $\mathbb{S}^{2} \times \mathbb{H}^{2} \times \mathbb{L}_{\pi}^{2}(0, T)$ which we equip with the norm $\|\cdot\|_{\mathcal{E}_{f}^{\beta}}$ defined by

$$
\|(Y, Z, \psi)\|_{\mathcal{E}_{f}^{\beta}}^{2}=\||Y|\|_{\beta}^{2}+\|Z\|_{\beta}^{2}+\|\psi\|_{\mathbb{L}_{\pi}^{2, \beta}}^{2}
$$

for all $(Y, Z, \psi) \in \mathbb{S}^{2} \times \mathbb{H}^{2} \times \mathbb{L}_{\pi}^{2}(0, T)$. After, we define an application $\Phi: \mathcal{E}_{f}^{\beta} \rightarrow \mathcal{E}_{f}^{\beta}$ as follows: for a given $(y, z, \varphi) \in \mathcal{E}_{f}^{\beta}$, we let $(Y, Z, \psi)=\Phi(y, z, \varphi)$ where $(Y, Z, \psi)$ the first three components of the solution to the RBSDE associated with driver $f:=f\left(t, y_{t}, z_{t}, \varphi_{t}\right)$ and with obstacle $\xi_{t}$. Let $(A, C)$ be the associated Mertens process, constructed as in lemma 3.5. The mapping $\Phi$ is well-defined by Lemma 3.5.

Let $(y, z, \varphi)$ and $\left(y^{\prime}, z^{\prime}, \varphi^{\prime}\right)$ be two elements of $\mathcal{E}_{f}^{\beta}$. We set $(Y, Z, \psi)=\Phi(y, z, \varphi)$ and $\left(Y^{\prime}, Z^{\prime}, \psi^{\prime}\right)=\Phi\left(y^{\prime}, z^{\prime}, \varphi^{\prime}\right)$. We also set $\widetilde{Y}=Y-Y^{\prime}, \widetilde{Z}=Z-Z^{\prime}, \widetilde{\psi}=\psi-\psi^{\prime}$, $\widetilde{y}=y-y^{\prime}, \widetilde{z}=z-z^{\prime}$ and $\widetilde{\varphi}=\varphi-\varphi^{\prime}$.

By the same argument that in the proof of Theorem 3.4 in Grigorova et al. (2015), in the Brownian filtration case. Let us prove that for a suitable choice of the parameter $\beta>0$, the mapping $\Phi$ is a contraction from the Banach space $\mathcal{E}_{f}^{\beta}$ into itself. Indeed, By applying Lemma 3.4, we have, for all $\epsilon>0$ and for all $\beta \geq \frac{1}{\epsilon^{2}}$ :

$$
\begin{aligned}
\|\widetilde{Y} \mid\|_{\beta}^{2}+\|\widetilde{Z}\|_{\beta}^{2}+\|\widetilde{\psi}\|_{\mathbb{L}_{\pi}^{2, \beta}}^{2} & \leq\|\widetilde{Y} \mid\|_{\beta}^{2}+\|\widetilde{Z}\|_{\beta}^{2}+\|\widetilde{M}\|_{\mathbb{M}_{\beta}^{2}}^{2}+\|\widetilde{\psi}\|_{\mathbb{L}_{\pi}^{2, \beta}}^{2} \\
& \leq \epsilon^{2}\left(5+16 c^{2}\right)\left\|f(t, y, z, \varphi)-f\left(t, y^{\prime}, z^{\prime}, \varphi^{\prime}\right)\right\|_{\beta}^{2} .
\end{aligned}
$$

By using the Lipschitz property of $f$ and the fact that $(a+b)^{2} \leq 2 a^{2}+2 b^{2}$, for all $(a, b) \in \mathbb{R}^{2}$, we obtain

$$
\left\|f(t, y, z, \varphi)-f\left(t, y^{\prime}, z^{\prime}, \varphi^{\prime}\right)\right\|_{\beta}^{2} \leq C_{K}\left(\|\widetilde{y}\|_{\beta}^{2}+\|\widetilde{z}\|_{\beta}^{2}+\|\widetilde{\varphi}\|_{\mathbb{L}_{\pi}^{2, \beta}}^{2}\right)
$$

where $C_{K}$ is a positive constant depending on the Lipschitz constant $K$ only. Thus, for all $\epsilon>0$ and for all $\beta \geq \frac{1}{\epsilon^{2}}$ we have:

$$
\||\widetilde{Y}|\|_{\beta}^{2}+\|\widetilde{Z}\|_{\beta}^{2}+\|\widetilde{\psi}\|_{\mathbb{L}_{\pi}^{2, \beta}}^{2} \leq \epsilon^{2} C_{K}\left(5+16 c^{2}\right)\left(\|\widetilde{y}\|_{\beta}^{2}+\|\widetilde{z}\|_{\beta}^{2}+\|\widetilde{\varphi}\|_{\mathbb{L}_{\pi}^{2, \beta}}^{2}\right) .
$$

The previous inequality, combined with (2) in Remark 3.6, gives

$$
\|\widetilde{Y} \mid\|_{\beta}^{2}+\|\widetilde{Z}\|_{\beta}^{2}+\|\widetilde{\psi}\|_{\mathbb{L}_{\pi}^{2, \beta}}^{2} \leq \epsilon^{2} C_{K}\left(5+16 c^{2}\right)(T+1)\left(\||\widetilde{y}|\|_{\beta}^{2}+\|\widetilde{z}\|_{\beta}^{2}+\|\widetilde{\varphi}\|_{\mathbb{L}_{\pi}^{2, \beta}}^{2}\right) .
$$

Thus, for $\epsilon>0$ such that $\epsilon^{2} C_{K}\left(5+16 c^{2}\right)(T+1)<1$ and $\beta>0$ such that $\beta \geq \frac{1}{\epsilon^{2}}$, the mapping $\Phi$ is a contraction. By the Banach fixed-point theorem, we get that $\Phi$ has a unique fixed point in $\mathcal{E}_{f}^{\beta}$. We thus have the existence and uniqueness of the solution to the RBSDE. 


\section{Application on dynamic risk measure and optimal stopping.}

4.1. On dynamic risk measure. In this subsection, we give an application of reflected BSDEs in dynamic risk measure. Indeed, define the following functional: for each stopping time $\tau \in \mathcal{T}_{0, T}$ and $\xi \in \mathbb{S}^{2}$. Set

$$
v(S)=-e s s \sup _{\tau \in \mathcal{T}_{S, T}} \mathcal{E}_{S, \tau}^{f}\left(\xi_{\tau}\right)
$$

where $S \in \mathcal{T}_{0, T}, v$ is the dynamic risk measure, $\xi_{T^{\prime}}\left(T^{\prime} \in[0, T]\right)$ is the gain of the position at time $T^{\prime}$ and $-\mathcal{E}_{t, T^{\prime}}^{f}\left(\xi_{T^{\prime}}\right)$ is the $f$-conditional expectation of $\xi_{\tau}$ modelling the risk at time $t$ where $t \in[0, T]$. We can show that the minimal risk measure $v$ defined by (4.1) coincides with $-Y$, where $Y$ is (the first component of) the solution to the reflected BSDE associated with driver $f$ and obstacle $\xi$. For this purpose, we can extend the results in Proposition A.5 and Theorem 4.2 in Grigorova et al. (2015) to our setting (see Aazizi and Ouknine, 2016 for more details).

4.2. On optimal stopping. We note also that we can show the existence of an $\varepsilon$ optimal stopping time, and that of the existence of an optimal stopping time under suitable assumptions on the barrier $\xi$ i.e. without right continuity of $\xi$, by extending the results of the second part of Grigorova et al. (2015) to our setting.

Let $(Y, Z, \psi, M, A, C)$ be the solution of the reflected BSDE with parameters $(f, \xi)$ as in definition 3.1, we have

$$
Y_{S}=e s s \sup _{\tau \in \mathcal{T}_{S, T}} \mathcal{E}_{S, \tau}^{f}\left(\xi_{\tau}\right)
$$

For each $S \in \mathcal{T}_{0, T}$ and $\varepsilon>0$, the stopping time $\tau_{S}^{\varepsilon}=\inf \left\{t \geq S, Y_{t} \leq \xi_{t}+\varepsilon\right\}$ is a $(C \varepsilon)$-optimal for 4.2 where $C$ is a constant which depends only on $T$ and the Lipschitz constant $K$ of $f$ :

$$
Y_{S} \leq \mathcal{E}_{S, \tau_{S}^{\varepsilon}}^{f}\left(\xi_{\tau_{S}^{\varepsilon}}\right)+C \varepsilon, \quad \text { a.s. }
$$

Under our assumption on $\xi$ and $f$, we can prove that for each $S \in \mathcal{T}_{0, T}$ and $\widehat{\tau} \in \mathcal{T}_{S, T}$, the stopping time $\widehat{\tau}$ is $S$-optimal. i.e.

$$
Y_{\widehat{\tau}}=\mathcal{E}_{S, \widehat{\tau}}^{f}\left(\xi_{\widehat{\tau}}\right), \text { a.s. }
$$

(see Theorem 4.2 and Proposition 4.3 in Grigorova et al., 2015).

Finally, under an additional assumption of left-upper semicontinuity (l.u.s.c) of $\xi$ in $\mathbb{S}^{2}$, the first time when the value process $Y$ hits $\xi$ is optimal: if $\tau_{S}^{*}=\inf \{u \geq$ $\left.S, Y_{u}=\xi_{u}\right\}, \xi$ is r.u.s.c and l.u.s.c in $\mathbb{S}^{2}$ and $(Y, Z, \psi, M, A, C)$ is the solution of the reflected BSDE of definition 3.1, the stopping time $\tau_{S}^{*}$ is optimal that is

$$
Y_{S}=\mathcal{E}_{S, \tau_{S}^{*}}^{f}\left(\xi_{\tau_{S}^{*}}\right), \quad \text { a.s. }
$$

(see Proposition 4.2 in Grigorova et al., 2015).

\section{Acknowledgements}

The authors would like to thank the referee for the careful reading of the paper and highly appreciate the comments and suggestions, which significantly contributed to improving the quality of the paper. 


\section{References}

S. Aazizi and Y. Ouknine. Strong envelope and strong supermartingale: application to reflected backward stochastic differential equation. ArXiv Mathematics eprints (2016). arXiv: 1112.0255.

J.-M. Bismut. Conjugate convex functions in optimal stochastic control. J. Math. Anal. Appl. 44, 384-404 (1973). MR0329726.

J.-M. Bismut. Théorie probabiliste du contrôle des diffusions. Mem. Amer. Math. Soc. 4 (167), xiii+130 (1976). MR0453161.

S. Crépey and A. Matoussi. Reflected and doubly reflected BSDEs with jumps: a priori estimates and comparison. Ann. Appl. Probab. 18 (5), 2041-2069 (2008). MR2462558.

C. Dellacherie and P.-A. Meyer. Probabilités et potentiel: Théorie des Martingales. Chapitres $V$ à VIII, volume 1385 of Actualités Scientifiques et Industrielles [Current Scientific and Industrial Topics]. Hermann, Paris, revised edition (1980). ISBN 2-7056-1385-4. MR566768.

N. El Karoui. Les aspects probabilistes du contrôle stochastique. In Ninth Saint Flour Probability Summer School-1979 (Saint Flour, 1979), volume 876 of Lecture Notes in Math., pages 73-238. Springer, Berlin-New York (1981). MR637471.

N. El Karoui, C. Kapoudjian, E. Pardoux, S. Peng and M. C. Quenez. Reflected solutions of backward SDE's, and related obstacle problems for PDE's. Ann. Probab. 25 (2), 702-737 (1997a). MR1434123.

N. El Karoui, S. Peng and M. C. Quenez. Backward stochastic differential equations in finance. Math. Finance 7 (1), 1-71 (1997b). MR1434407.

E. H. Essaky. Reflected backward stochastic differential equation with jumps and RCLL obstacle. Bull. Sci. Math. 132 (8), 690-710 (2008). MR2474488.

L. I. Gal'čuk. Optional martingales. Mat. Sb. (N.S.) 112(154) (4(8)), 483-521 (1980). ISSN 0368-8666. MR587036.

M. Grigorova, P. Imkeller, E. Offen, Y. Ouknine and M.-C. Quenez. Reflected BSDEs when the obstacle is not right-continuous and optimal stopping. ArXiv Mathematics e-prints (2015). arXiv: 1504.06094.

S. Hamadène. Reflected BSDE's with discontinuous barrier and application. Stoch. Stoch. Rep. 74 (3-4), 571-596 (2002). MR1943580.

S. Hamadène and Y. Ouknine. Reflected backward stochastic differential equation with jumps and random obstacle. Electron. J. Probab. 8, no. 2, 20 (2003). MR1961164.

S. Hamadène and Y. Ouknine. Reflected backward SDEs with general jumps. Theory Probab. Appl. 60 (2), 263-280 (2016). MR3568776.

J. Jacod and A. N. Shiryaev. Limit theorems for stochastic processes, volume 288 of Grundlehren der Mathematischen Wissenschaften [Fundamental Principles of Mathematical Sciences]. Springer-Verlag, Berlin, second edition (2003). ISBN 3-540-43932-3. MR1943877.

M. Kobylanski and M.-C. Quenez. Optimal stopping time problem in a general framework. Electron. J. Probab. 17, no. 72, 28 (2012). MR2968679.

E. Lenglart. Tribus de Meyer et théorie des processus. In Seminar on Probability, XIV (Paris, 1978/1979) (French), volume 784 of Lecture Notes in Math., pages 500-546. Springer, Berlin (1980). MR580151.

A. Nikeghbali. An essay on the general theory of stochastic processes. Probab. Surv. 3, 345-412 (2006). MR2280298. 
É. Pardoux and S. Peng. Backward stochastic differential equations and quasilinear parabolic partial differential equations. In Stochastic partial differential equations and their applications (Charlotte, NC, 1991), volume 176 of Lect. Notes Control Inf. Sci., pages 200-217. Springer, Berlin (1992). MR1176785.

É. Pardoux and S. G. Peng. Adapted solution of a backward stochastic differential equation. Systems Control Lett. 14 (1), 55-61 (1990). MR1037747.

S. Peng. Monotonic limit theorem of BSDE and nonlinear decomposition theorem of Doob-Meyer's type. Probab. Theory Related Fields 113 (4), 473-499 (1999). MR1717527.

P. Protter. Stochastic integration and differential equations, volume 21 of Applications of Mathematics (New York). Springer-Verlag, Berlin (1990). ISBN 3-54050996-8. MR1037262.

M.-C. Quenez and A. Sulem. Reflected BSDEs and robust optimal stopping for dynamic risk measures with jumps. Stochastic Process. Appl. 124 (9), 3031-3054 (2014). MR3217432. 